\title{
The Lingering Anomalous Secular Increase of the Eccentricity of the Orbit of the Moon: Further Attempts of Explanations of Cosmological Origin
}

\section{Lorenzo Iorio}

Italian Ministry of Education, University and Research (M.I.U.R.)-Education, Fellow of the Royal Astronomical Society (F.R.A.S.), Viale Unità di Italia 68, Bari (BA) 70125, Italy;

E-Mail: lorenzo.iorio@libero.it; Tel.: +39-329-239-9167

Received: 18 April 2014 / Accepted: 25 April 2014 /

Published: 2 May 2014

\begin{abstract}
New analyses of extended data records collected with the Lunar Laser Ranging (LLR) technique performed with improved tidal models were not able to resolve the issue of the anomalous rate $\dot{e}$ of the eccentricity $e$ of the orbit of the Moon, which is still in place with a magnitude of $\dot{e}=(5 \pm 2) \times 10^{-12} \mathrm{yr}^{-1}$. Some possible cosmological explanations are offered in terms of the post-Newtonian effects of the cosmological expansion and of the slow temporal variation of the relative acceleration rate $\ddot{S} S^{-1}$ of the cosmic scale factor $S$. None of them is successful since their predicted secular rates of the lunar eccentricity are too small by several orders of magnitude.
\end{abstract}

Keywords: experimental studies of gravity; experimental tests of gravitational theories; modified theories of gravity; ephemerides, almanacs, and calendars

Classification: PACS 04.80.-y; 04.80.Cc; 04.50.Kd; 95.10.Km

\section{Introduction}

In 2009, Williams and Boggs [1] reported an anomalous secular rate of the eccentricity $e$ of the orbit of the Moon:

$$
\dot{e}=(9 \pm 3) \times 10^{-12} \mathrm{yr}^{-1}
$$

They fit the dynamical models of the DE421 ephemerides [2] to a record of ranges collected with the Lunar Laser Ranging (LLR) technique from March 1970 to November 2008. The effect of Equation (1) 
is anomalous in the sense that it is in excess with respect to the eccentricity rate predicted with the tidal models of the DE421 ephemerides. Anderson and Nieto [3] included Equation (1) among the current astrometric anomalies in the Solar System. Some more or less sound attempts to find an explanation of Equation (1) in terms of non-standard physics have been performed so far [4-8].

Recently, Williams et al. [9] extended their analysis of the LLR data from March 1970 to April 2013 by using the new DE430 ephemerides [10] with improved tidal models. As a result, the anomalous eccentricity rate of the lunar orbit, although reduced with respect to Equation (1), did not disappear, amounting now to (as remarked in [9], the rate of Equation (2) exhibited a low correlation with other solved-for estimated parameters):

$$
\dot{e}=(5 \pm 2) \times 10^{-12} \mathrm{yr}^{-1}
$$

In this paper, we propose to look for further possible physical mechanisms able to explain the lingering anomalous lunar eccentricity rate.

\section{Ruling Out Some Possible Mechanisms of Cosmological Origin}

At the Newtonian level, the cosmological expansion induces a Hooke-type two-body acceleration proportional to the binary's separation $r$ through an "elastic" constant $\mathcal{K}$ given by the relative acceleration rate $\ddot{S} S^{-1}$ of the cosmic scale factor $S$; see, e.g., [11] and references therein. Such an acceleration, proportional to the square of the Hubble parameter $H$, affects neither the shape nor the size of the orbit of a localized binary system, as it was calculated by several authors with a variety of different approaches [12-16].

At the first post-Newtonian (pN) level, a velocity-dependent acceleration linear in $H$ occurs [17]: in principle, it does secularly change both the semimajor axis $a$ and the eccentricity $e$ of the orbit of a test particle moving about a central body of mass $M$ [18]. The resulting eccentricity rate, averaged over one orbital period $P_{\mathrm{b}}$ of the test particle, is [18]:

$$
\dot{e}^{(H \mathrm{pN})}=\frac{4 H_{0} a^{2} n_{\mathrm{b}}^{2} \sqrt{1-e^{2}}\left(-1+\sqrt{1-e^{2}}\right)}{c^{2} e}
$$

where $c$ is the speed of light in vacuum, $H_{0}$ is the value of the Hubble parameter at the present epoch, and ( $G$ is the Newtonian constant of gravitation) $n_{\mathrm{b}}=\sqrt{G M a^{-3}}=2 \pi P_{\mathrm{b}}^{-1}$ is the Keplerian mean motion. In the case of the Earth-Moon system, by using the latest determination for the Hubble parameter from the Planck mission [19] $H_{0}=6.88 \times 10^{-11} \mathrm{yr}^{-1}$, Equation (3) yields a (negative) lunar eccentricity rate as little as:

$$
\dot{e}^{(H \mathrm{pN})}=-1 \times 10^{-22} \mathrm{yr}^{-1}
$$

Apart from the sign, Equation (4) is 10 orders of magnitude smaller than Equation (2).

Recently, in [20] it has been pointed out that a slow temporal variation of $\ddot{S} S^{-1}$ affects the local dynamics of a two-body system by secularly changing both $a$ and $e$. In the case of matter-dominated epochs with Dark Energy, it turns out that, to first order in the power expansion of $\ddot{S} S^{-1}$, the mean eccentricity rate is [20]:

$$
\dot{e}^{\left(\Omega_{\Lambda}\right)}=\frac{(4+e)\left(-1+e^{2}\right) \mathcal{K}_{1}}{4 n_{\mathrm{b}}^{2}}
$$


where

$$
\mathcal{K}_{1} \doteq \frac{3}{2} H_{0}^{3} \Omega_{\Lambda}^{3 / 2} \operatorname{coth}\left(\frac{3}{2} H_{0} t_{0} \sqrt{\Omega_{\Lambda}}\right) \operatorname{csch}^{2}\left(\frac{3}{2} H_{0} t_{0} \sqrt{\Omega_{\Lambda}}\right)
$$

in which $t_{0}$ is the present epoch, while $\Omega_{\Lambda}$ is the Dark Energy density normalized to the critical energy density. The latest values $t_{0}=13.817 \mathrm{Gyr}, \Omega_{\Lambda}=0.685$ from Planck [19] and Equations (5) and (6) yield for the Moon:

$$
\dot{e}^{\left(\Omega_{\Lambda}\right)}=-2 \times 10^{-35} \mathrm{yr}^{-1}
$$

which is 23 orders of magnitude smaller that the observed anomalous eccentricity rate.

Thus, we can rule out any potentially viable explanation of cosmological origin for Equation (2).

\section{Conclusions}

As a result of the latest LLR data analysis performed with improved tidal models, it turned out that the anomalous eccentricity rate of the lunar orbit is still lingering, and amounts to $\dot{e}=(5 \pm 2) \times 10^{-12} \mathrm{yr}^{-1}$.

The LLR analysts seem convinced that, sooner or later, a better understanding of the intricate geophysical processes of tidal origin taking place in the interiors of our planet and of its satellite will be able to fully accommodate the orbital anomaly. As such, they will continue to look for conventional causes for the anomalous eccentricity rate of the Moon.

Nonetheless, as a complementary approach, the search for causes residing outside the Earth and the Moon themselves is worthy of being pursued. If unsuccessful, it could also indirectly strengthen the relevance of the efforts towards an explanation in terms of standard physics. In this respect, it has been shown that neither the cosmological expansion at the first post-Newtonian level nor the slow temporal variation of the relative acceleration rate of the cosmic scale factor are able to explain the anomalous eccentricity increase because they induce long-term rates of change of the Moon's eccentricity too small by several orders of magnitude. Such a further negative result adds to the previous series of failed attempts to find sound non-tidal explanations in the literature so far.

\section{Conflicts of Interest}

The author declare no conflict of interest.

\section{References}

1. Williams, J.G.; Boggs, D.H. Lunar Core and Mantle. What Does LLR See? In Proceedings of the 16th International Workshop on Laser Ranging, Poznan, Poland, 13-17 October 2008.

2. Williams, J.G.; Boggs, D.H.; Folkner, W.M. DE421 Lunar Orbit, Physical Librations, and Surface Coordinates. Available online: ftp://ssd.jpl.nasa.gov/pub/eph/planets/ioms/de421_moon_coord_ iom.pdf (accessed on 1 May 2014).

3. Anderson, J.D.; Nieto, M.M. Astrometric solar-system anomalies. Proc. Int. Astron. Union 2010, $5,189-197$.

4. Iorio, L. On the anomalous secular increase of the eccentricity of the orbit of the Moon. Mon. Not. R. Astron. Soc. 2011, 415, 1266-1275. 
5. Iorio, L. An Empirical Explanation of the Anomalous Increases in the Astronomical Unit and the Lunar Eccentricity. Astron. J. 2011, 142, doi:10.1088/0004-6256/142/3/68.

6. Ziefle, R.G. Explanation of the anomalous secular increase of the moon orbit eccentricity by the new theory of gravitation (NTG). Phys. Essays 2013, 26, 82-85.

7. Acedo, L. Anomalous post-Newtonian terms and the secular increase of the astronomical unit. $A d v$. Space Res. 2013, 52, 1297-1303.

8. Acedo, L. A phenomenological variable speed of light theory and the secular increase of the astronomical unit. Phys. Essays 2013, 26, 567-573.

9. Williams, J.G.; Turyshev, S.G.; Boggs, D.H. The past and present Earth-Moon system: The speed of light stays steady as tides evolve. Planet. Sci. 2014, 3, doi:10.1186/s13535-014-0002-5.

10. Folkner, W.M.; Williams, J.G.; Boggs, D.H.; Park, R.S.; Kuchynka, P. The Planetary and Lunar Ephemerides DE430 and DE431. Interplanet. Netw. Prog. Rep. 2014, 196, 1-81.

11. Carrera, M.; Giulini, D. Influence of global cosmological expansion on local dynamics and kinematics. Rev. Mod. Phys. 2010, 82, 169-208.

12. Cooperstock, F.I.; Faraoni, V.; Vollick, D.N. The Influence of the Cosmological Expansion on Local Systems. Astrophys. J. 1998, 503, doi:10.1086/305956.

13. Mashhoon, B.; Mobed, N.; Singh, D. Tidal dynamics in cosmological spacetimes. Class. Quantum Gravity 2007, 24, 5031-5046.

14. Sereno, M.; Jetzer, P. Evolution of gravitational orbits in the expanding universe. Phys. Rev. D 2007, 75, doi:10.1103/PhysRevD.75.064031.

15. Adkins, G.S.; McDonnell, J.; Fell, R.N. Cosmological perturbations on local systems. Phys. Rev. D 2007, 75, doi:10.1103/PhysRevD.75.064011.

16. Nandra, R.; Lasenby, A.N.; Hobson, M.P. The effect of an expanding universe on massive objects. Mon. Not. R. Astron. Soc. 2012, 422, 2945-2959.

17. Kopeikin, S.M. Celestial ephemerides in an expanding universe. Physical Review D 2012, 86, doi:10.1103/PhysRevD.86.064004.

18. Iorio, L. Local cosmological effects of the order of $\mathrm{H}$ in the orbital motion of a binary system. Mon. Not. R. Astron. Soc. 2013, 429, 915-922.

19. Planck Collaboration; Ade, P.A.R.; Aghanim, N.; Armitage-Caplan, C.; Arnaud, M.; Ashdown, M.; Atrio-Barandela, F.; Aumont, J.; Baccigalupi, C.; Banday, A.J.; et al. Planck 2013 results. XVI. Cosmological parameters. ArXiv E-Prints, 2013, arXiv:1303.5076.

20. Iorio, L. Two-Body Orbit Expansion Due to Time-Dependent Relative Acceleration Rate of the Cosmological Scale Factor. Galaxies 2014, 2, 13-21.

(c) 2014 by the author; licensee MDPI, Basel, Switzerland. This article is an open access article distributed under the terms and conditions of the Creative Commons Attribution license (http://creativecommons.org/licenses/by/3.0/). 